\title{
Sampling effects on the Empirical Mode Decomposition
}

\author{
Gabriel Rilling $\quad$ Patrick Flandrin \\ Laboratoire de Physique (UMR 5672 CNRS), \\ Ecole Normale Supérieure de Lyon, \\ 46 allée d'Italie, 69364 Lyon Cedex 07 France. \\ E-mail: \{grilling,flandrin\}@ens-lyon.fr
}

\begin{abstract}
Standard exposition of Empirical Mode Decomposition (EMD) is usually done within a continuous-time setting whereas, in practice, the effective implementation always operates in discrete-time. The purpose of this contribution is to summarize a number of results aimed at quantifying the influence of sampling on EMD. The idealized case of a sampled pure tone is first considered in detail and a theoretical model is proposed for upper bounding the approximation error due to finite sampling rates. A more general approach is then discussed, based on the analysis of the nonlinear operator that underlies the EMD (one step) sifting process. New explicit, yet looser, bounds are obtained this way, whose parameters can be estimated directly from the analyzed signal. Theoretical predictions are compared to simulation results in a number of well-controlled numerical experiments.
\end{abstract}

\section{Introduction}

In most expositions of "Empirical Mode Decomposition" (EMD), it is implicitly assumed that the considered signals are given in continuous-time and, indeed, the rationale underlying EMD is more intuitive this way [1]. In practice however, EMD is usually implemented in discrete-time and applied to digital time series (see, e.g., [3,7]), either because the analyzed data is intrinsically discrete, or because it results from the sampling of some underlying continuous-time process. Considering a continuous-time signal, the sampling step which is required prior applying EMD is expected to affect the resulting decomposition in some way, and it is therefore the purpose of this paper to address some of the issues raised by sampling in the context of EMD, so as to help in a well-controlled (and, hopefully, robust) use of the technique in practical situations. More precisely, the paper is organized as follows. Section 2 provides a detailed analysis of the influence of sampling in the simplified case of a single tone. Under a number of assumptions that are critically examined, an upper bound is derived for the approximation due to finite sampling rates, and the relevance of the theoretical model is supported by numerical simulations. The analysis is further generalized beyond tones in Section 3, on the basis of a study of the elementary sifting operator involved in the EMD. This results again in a predicted bound for the error, that it shown to depend on parameters that can be estimated from the data. A set of numerical simulations with synthetic signals is then performed for assessing the performance of the theoretical upper bound model. 

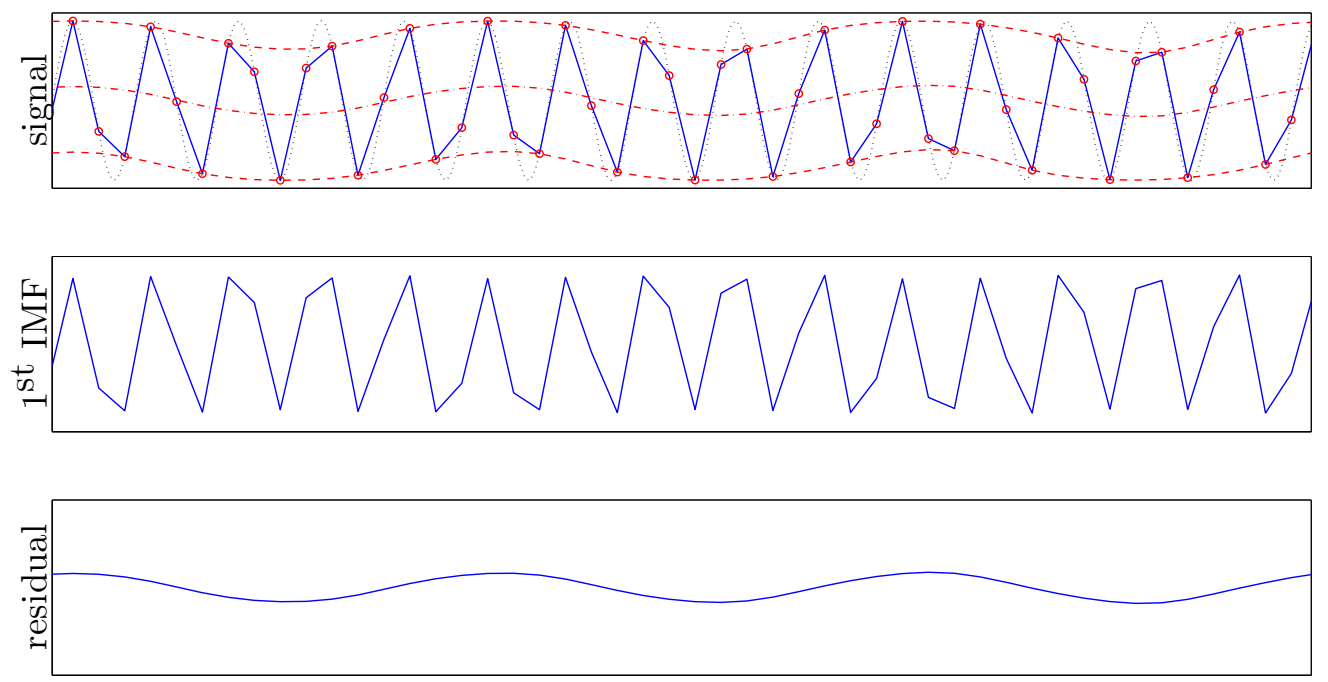

Figure 1: EMD of a tone-Qualitative influence of sampling. The analyzed tone is plotted in dotted line and its sampled version in full blue line (top diagram). Due to the finite (though admissible in Shannon's sense) sampling rate, the upper and lower envelopes as well as their local mean (plotted in red dashed lines) are oscillating. As a result of this oscillation, the 1st extracted IMF (middle diagram) does not exhaust the description of the signal as it would be expected for a continuous-time tone, and a non-zero residual oscillation is introduced (bottom diagram).

\section{EMD of a tone}

Let us start with the simplest example of a continuous-time tone, i.e., a waveform $x(t)$ defined as the sinusoidal, unit period, signal:

$$
x(t)=\cos 2 \pi t .
$$

In this idealized situation, the analyzed signal fulfils — by construction - all the requirements for being an "Intrinsic Mode Function" (IMF) [1], and EMD is in this case expected to act as the identity operator, with the signal itself as the unique output IMF and no residual. Whereas this interpretation obviously holds true in continuous-time, it turns out that the picture is dramatically changed when sampling enters the play. In fact, EMD is basically constructed on signal extrema, and the extrema of a sampled signal generally differ from those of its continuous-time counterpart: it follows that the local mean (defined as the mean of the envelopes interpolating the extrema) may present some sampling related artefacts, as illustrated in Figure 1.

\subsection{Sampling error as a function of the sampling frequency}

In order to evaluate the influence of sampling, we introduce sampled versions of (1) as:

$$
x_{f_{s}, \varphi}[n]=x\left(\frac{n}{f_{s}}+\varphi\right)=\cos \left(\frac{2 \pi}{f_{s}} n+2 \pi \varphi\right)
$$

with $n \in \mathbb{Z}$, where $f_{s}$ and $\varphi$ stand for the sampling frequency and phase, respectively. Since the original signal (1) is already an IMF, we then propose to evaluate the influence of the sampling 
parameters $f_{s}$ and $\varphi$ by using as an error criterion the simple $\left(l_{1}\right)$ discrepancy function:

$$
e_{x}\left(f_{s}, \varphi\right)=\frac{\sum_{n=1}^{N}\left|x_{f_{s}, \varphi}[n]-d_{f_{s}, \varphi}[n]\right|}{\sum_{n=1}^{N}\left|x_{f_{s}, \varphi}[n]\right|},
$$

where $d_{f_{s}, \varphi}[n]$ stands for the first IMF computed from the discrete-time version (2) of the continuoustime tone (1). We will also define and use another quantity $\bar{e}_{x}\left(f_{s}\right)$, which is the phase averaged version of $e_{x}\left(f_{s}, \varphi\right)$ :

$$
\bar{e}_{x}\left(f_{s}\right)=\mathbb{E}_{\varphi}\left\{e_{x}\left(f_{s}, \varphi\right)\right\}=\int_{0}^{1} e_{x}\left(f_{s}, \varphi\right) d \varphi
$$

with $\varphi$ a random variable uniformly distributed over the interval $[0,1[$.

\subsection{Bounding the sampling error}

The influence of sampling on the EMD of a pure tone has already been partly considered in previous publications [3-6], and it has been observed (from simulation experiments) that the criterion (3) admits an upper bound inversely proportional to the square of the sampling frequency ${ }^{1}$. The purpose of this section is to justify this observed behaviour. More precisely, we will prove that:

$$
e_{x}\left(f_{s}, \varphi\right) \leq \frac{\pi}{4}\left(1-\cos \frac{\pi}{f_{s}}\right) \leq \frac{\pi^{3}}{8 f_{s}^{2}}
$$

the first bound being furthermore tight when $\varphi=0$ and $f_{s}=2 k+1, k \in \mathbb{N}$.

In order to do so, we will have to make some assumptions, which consist in the following three approximations:

1. The upper envelope interpolating the maxima takes values between the smallest maximum and 1. Similarly, the lower envelope takes values between -1 and the greatest minimum.

2. The first IMF is obtained through a unique sifting step.

3. The $l_{1}$-norm of $x_{f_{s}, \varphi}[n]$ is exactly $2 N / \pi$.

The first approximation allows us to control the value of the envelopes by just considering the extrema. Actually, this approximation can also be exact depending on the interpolation used to compute the envelopes. As a matter of fact, this is the case for linear interpolation and for the built-in MATLAB pchip (a.k.a. cubic) interpolation. Unfortunately, it is only an approximation for the cubic spline interpolation, which is by far the most commonly used. Nevertheless, this approximation holds well in the specific case of sinusoidal signals.

The second approximation is a bit more questionable. In fact, it generally takes more than one sifting step to obtain an admissible IMF. However, the first sifting step is always the most

\footnotetext{
${ }^{1}$ In fact, the experiments conducted in [3,4] adopted a reversed perspective as compared to the approach followed here: the sampling frequency was indeed kept fixed (and arbitrarily set to unity) and the frequency of the analyzed tone was varied. It followed that the error measure was evaluated as a function of the tone frequency, with an observed upper bound proportional to its square.
} 
important one, and the following steps just improve slightly the final result. It has nevertheless to be noticed that this may not be the case for any input signal: for some signals indeed, the first sifting step can reveal new extrema and, therefore, the next sifting step may be as important as the first one. In the specific case of sinusoidal signals, there are however no new extrema revealed by any sifting step, and neglecting the sifting steps after the first one is therefore a reasonable hypothesis.

Finally, the third approximation is nearly exact for almost all sampling frequencies provided there is a large number of periods in the signal. The only exceptions occur for sampling frequencies that are simple rational numbers but, even in these cases, the discrepancy is rather small.

Assuming that the three above approximations hold, the proof of (5) proceeds as follows. Using first the third hypothesis, we can write (3) as:

$$
\begin{aligned}
e_{x}\left(f_{s}, \varphi\right) & =\frac{\sum_{n=1}^{N}\left|x_{f_{s}, \varphi}[n]-d_{f_{s}, \varphi}[n]\right|}{\sum_{n=1}^{N}\left|x_{f_{s}, \varphi}[n]\right|} \\
& =\frac{\pi}{2 N} \sum_{n=1}^{N}\left|m_{f_{s}, \varphi}[n]\right|,
\end{aligned}
$$

where $m_{f_{s}, \varphi}[n]$ is the local mean of $x_{f_{s}, \varphi}[n]$, which is also the mean of the envelopes $e_{\min }[n]$ and $e_{\max }[n]$ thanks to the second hypothesis. Using then the first hypothesis, we know that $e_{\max }[n]$ is positive and $e_{\min }[n]$ negative. Thus,

$$
\begin{aligned}
\left|m_{f_{s}, \varphi}[n]\right| & =\left|\frac{e_{\max }[n]+e_{\min }[n]}{2}\right| \\
& =\frac{\left\|e_{\max }[n]|-| e_{\min }[n]\right\|}{2} \\
& \leq \frac{1-\alpha}{2},
\end{aligned}
$$

where $-\alpha$ is an upper bound for the values of the minima or, alternatively, $\alpha$ is a lower bound for the maxima. The value of $\alpha$ is rather easy to obtain as it corresponds to the case where two consecutive sampling points hit the continuous-time sinusoid symetrically with respect to one of its extrema. We get therefore $\alpha=\cos \left(\pi / f_{s}\right)$ and, using finally the common formula "cos $u \geq 1-u^{2} / 2$ ", we obtain the last part of the desired result (5).

The obtained upper bound is furthermore reached for sampling frequencies $f_{s}=2 k+1, k \in \mathbb{N}$ and phase $\varphi=0$. In this case indeed, the upper and lower envelopes are constants with respective values 1 and $-\cos \left(\pi / f_{s}\right)$, and the $\pi^{3} / 8$ coefficient in the $f_{s}^{-2}$ bound is thus optimal. This concludes the proof.

Figure 2 (left diagram) illustrates the behaviour of the actual error (3) as a function of the sampling frequency, as well as the effectiveness of the upper bound (5). 

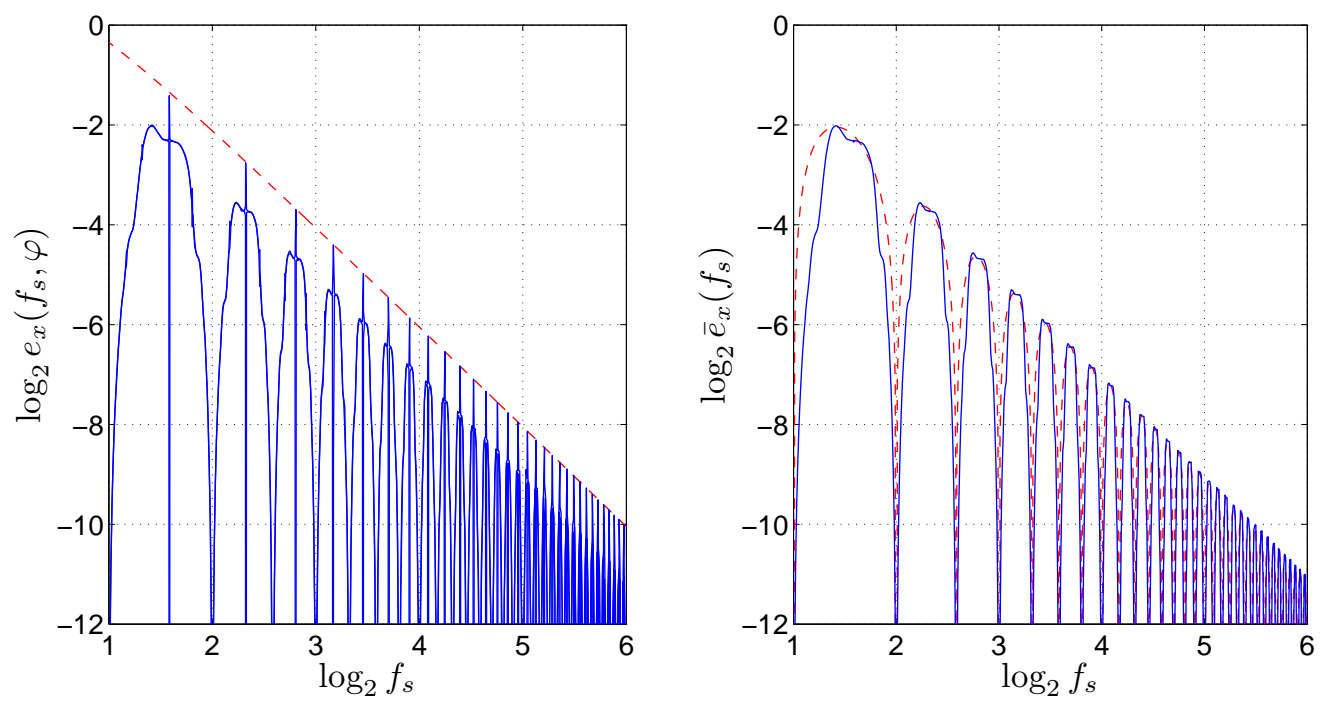

Figure 2: EMD of a tone-Quantitative influence of sampling. The left diagram displays (full blue lines) the actual maximum and minimum (with respect to the phase) error (3) as a function of the sampling frequency in the case of a tone with $N=2048$ data points, the superimposed dashed red line corresponding to the (tightest) upper bound given by (5). The right diagram displays the same way (full blue line) the associated phase-averaged error (4), the superimposed dashed red curve corresponding to the model (10).

\subsection{A model for the phase-averaged sampling error}

Based on the same set of hypotheses, we can propose for the phase-averaged sampling error a model which is closer to the observed experimental results. Unlike the previous analysis, this model does not give, per se, an upper bound for $\bar{e}_{x}\left(f_{s}\right)$ but rather an order of magnitude for it and, thus, for $e_{x}\left(f_{s}, \varphi\right)$. An advantage of this new model (which, as for the upper bound model, is only based on some considerations about the extrema of the sampled signal) is however its ability to describe the overall shape of the sampling error and, in particular, to justify its decay around sampling frequencies that match even integers.

Let us first notice that the sampling phase being a random variable uniformly distributed over $\left[0,1\left[\right.\right.$, the process $x_{f_{s}, \varphi}[n]$ is stationary. If we ignore boundary conditions issues, we can assume that $d_{f_{s}, \varphi}[n]$ and $m_{f_{s}, \varphi}[n]$ are stationary processes as well. Therefore, using (6), we can simplify the expression of $\bar{e}_{x}\left(f_{s}\right)$ and get, for any $n$ :

$$
\bar{e}_{x}\left(f_{s}\right)=\frac{\pi}{2} \mathbb{E}_{\varphi}\left\{\left|m_{f_{s}, \varphi}[n]\right|\right\}
$$

The next step is therefore to estimate locally the value of the local mean of the signal. At this end, the main idea is to approximate the values of the envelopes by the values of the nearest extrema. It follows that the absolute value of the local mean can be approximated by:

$$
\left|m_{f_{s}, \varphi}[n]\right| \approx \frac{1}{2}\left|\cos \left(\frac{2 \pi}{f_{s}} n_{\max }+2 \pi \varphi\right)+\cos \left(\frac{2 \pi}{f_{s}} n_{\min }+2 \pi \varphi\right)\right|
$$


where $n_{\max }\left(\right.$ resp. $n_{\min }$ ) refers to the index of the nearest maximum (resp. minimum).

Knowing that a maximum of the sampled signal is distant from the corresponding maximum of its continuous-time counterpart by at most half a sampling period, there exists necessarily an integer $k$ such that

$$
\varphi_{\max } \equiv \frac{n_{\max }}{f_{s}}+\varphi-k \in\left[-\frac{1}{2 f_{s}}, \frac{1}{2 f_{s}}\right] .
$$

Moreover, if $\varphi$ varies uniformly over $\left[0,1\left[\right.\right.$, so does $\varphi_{\max }$ over $\left[-1 /\left(2 f_{s}\right), 1 /\left(2 f_{s}\right)[\right.$ and, therefore:

$$
\mathbb{E}_{\varphi}\left\{\left|m_{f_{s}, \varphi}[n]\right|\right\} \approx \frac{1}{2} \mathbb{E}_{\psi}\left\{\left|\cos \psi+\cos \left(\frac{2 \pi \tilde{n}(\psi)}{f_{s}}+\psi\right)\right|\right\},
$$

where $\psi$ is a random variable uniformly distributed over $\left[-\pi / f_{s}, \pi / f_{s}[\right.$ and $\tilde{n}(\psi)$ refers to the index of the first minimum of $\cos \left(2 \pi n / f_{s}+\psi\right)$. If we let $K$ be the integer part of $f_{s} / 2$ and $\tilde{\psi}=\pi-(2 K+1) \pi / f_{s}$, we can express $\tilde{n}(\psi)$ as follows:

$$
\tilde{n}(\psi)= \begin{cases}K & \text { if } \psi>\tilde{\psi} \\ K+1 & \text { if } \psi<\tilde{\psi}\end{cases}
$$

Replacing $\tilde{n}(\psi)$ by its value in (8) yields:

$$
\begin{aligned}
& \mathbb{E}_{\varphi}\left\{\left|m_{f_{s}, \varphi}\right|\right\} \approx \frac{f_{s}}{4 \pi} \int_{-\pi / f_{s}}^{\pi / f_{s}}\left|\cos \psi+\cos \left(\frac{2 \pi \tilde{n}(\psi)}{f_{s}}+\psi\right)\right| d \psi \\
& \approx \frac{f_{s}}{4 \pi}\left[\int_{\tilde{\psi}}^{\pi / f_{s}}\left|\cos \psi+\cos \left(\frac{2 \pi K}{f_{s}}+\psi\right)\right| d \psi\right. \\
&\left.\quad+\int_{-\pi / f_{s}}^{\tilde{\psi}}\left|\cos \psi+\cos \left(\frac{2 \pi(K+1)}{f_{s}}+\psi\right)\right| d \psi\right] .
\end{aligned}
$$

Plugging this result into (7), we get after some algebra:

$$
\bar{e}_{x}\left(f_{s}\right) \approx \frac{f_{s}}{2}\left[\cos \left(\frac{K \pi}{f_{s}}\right)\left(1-\sin \left(\frac{(K+1) \pi}{f_{s}}\right)\right)+\cos \left(\frac{(K+1) \pi}{f_{s}}\right)\left(\sin \left(\frac{K \pi}{f_{s}}\right)-1\right)\right] .
$$

Finally, the above result can be greatly simplified by the use of the common approximations " $\sin u \approx u$ " and " $1-\cos u \approx u^{2} / 2$ for small $u$ 's. If we assume that $\pi / f_{s} \ll 1$, we have then $\left|\pi / 2-K \pi / f_{s}\right| \ll 1$ too and, therefore, (9) reduces to:

$$
\bar{e}_{x}\left(f_{s}\right) \approx \frac{\pi^{3}}{16 f_{s}^{2}}\left(2(K+1)-f_{s}\right)\left(f_{s}-2 K\right) .
$$

As it can be seen from this last equation, the model results in a parabolic approximation of $\bar{e}_{x}\left(f_{s}\right)$ on each sampling frequency interval of the form $\{[2 k, 2(k+1)], k \in \mathbb{N}\}$, thus justifying the decay of $\bar{e}_{x}\left(f_{s}\right)$ around frequencies that match even numbers. Moreover, the model accounts for the " $1 / f_{s}^{2}$ " tendency reported above, as evidenced by the prefactor, and it is also equal to $\bar{e}_{x}\left(f_{s}\right)$ for every integer sampling frequency ${ }^{2}$.

The model (9) and its approximation (10) are plotted in Figure 2 (right diagram). It is worth noticing that the last approximation holds well for the whole frequency range.

\footnotetext{
${ }^{2}$ Unlike what is said in [4], the corresponding model in this paper is also exact under the very same conditions, up to a missing $\sqrt{2}$ factor.
} 


\section{Generalization beyond tones}

To address properly the sampling issue, we first have to define some minimum requirements on the sampling parameters for a continuous-time signal to be processed by EMD. As extrema play a major role in the sifting operator, a natural requirement would be for a discrete-time signal to keep as many extrema as does its continuous-time counterpart. In fact, losing one extremum during the sampling process usually means losing a pair of maximum/minimum, which in turns means losing one local oscillation for the EMD. To ensure that there is no loss of extrema, the minimum requirement is for the sampling period to be at most one half of the minimum distance between extrema in the signal. In all the following, we will implicitly consider that this requirement is met. $^{3}$

\subsection{A bound on the sampling error for the elementary sifting operator}

Given a continuous-time signal $x(t)$ with a minimum distance $\Delta$ between its extrema, we will consider as before its discretized versions $\left\{x_{f_{s}, \varphi}[n]=x\left(n / f_{s}+\varphi\right), n \in \mathbb{Z}\right\}$, with the aim of characterizing the behaviour of their EMD as a function of the sampling frequency $f_{s}>2 / \Delta$. More particularly, we will be mainly interested in the deviation between the sampled IMFs and the theoretical, continuous-time, ones. When dealing with sinusoidal signals however, we based ourselves on three hypotheses (see Sect.2.2). Among these, the second one, saying that the first IMF is obtained through a unique sifting step, is a fair approximation for sinusoidal signals but becomes a really strong assumption for more general cases. Indeed, some extrema pairs are likely to appear after any iteration of the sifting process, thus compelling us to consider all the sifting steps. This unfortunately brings about two major issues to the analysis of the influence of sampling:

1. If an extrema pair appears at some point in the sifting process for a given set of sampling parameters, there is no guarantee that a similar extrema pair will ever appear for another set, even for same sampling frequencies and different phases. Moreover, the appearance of an extrema pair for a given set of sampling parameters seems rather unpredictable.

2. We generally don't know how many sifting steps are to be performed to extract the first IMF. Moreover, as this is generally decided by a test within the sifting loop, the precise number of iterations may depend on the analyzed discrete signal and therefore on the sampling parameters.

Because of these cumbersome issues, we will mainly focus on the effect of sampling on one sifting step only, or on what we refer to as "the sifting elementary operator" (thereafter denoted $\mathcal{S}$ )

\footnotetext{
${ }^{3}$ It is worth noticing that this requirement is completely independent from Shannon's sampling criterion for band-limited signals: indeed, a band-limited signal can have arbitrary close extrema, whatever its frequency band. For instance, $t \mapsto(1-\epsilon) \sin \epsilon f t-\epsilon \sin f t$ has two extrema at $\pm \sqrt{2 \epsilon} / f$ for $\epsilon \ll 1$.
} 
corresponding to the operation of subtracting to a signal the mean of its envelopes. More precisely, we will investigate the case of a simplified elementary operator for which we can derive an upper bound for the sampling effects. The obtained results will then be assessed by simulations using the original operator.

\subsubsection{Model}

We start off with analyzing the effects of sampling on the extrema. If $t_{0}$ is the position of a local maximum in $x(t)$, the condition $f_{s}>2 / \Delta$ ensures that there is also a maximum in $x_{f_{s}, \varphi}[n]$ for $n$ such that $\left|n / f_{s}+\varphi-t_{0}\right|<1 / f_{s}$, i.e., that the closest sampling point - either on the right or on the left — of the continuous-time maximum is a maximum for the sampled signal. More precisely, we can define the index of the extremum in the sampled signal as the one (or one of the two) such that $n / f_{s}+\varphi \in I_{0} \equiv\left[t_{0}-a, t_{0}+b\right]$ with $a, b>0, a+b=1 / f_{s}$ and $x\left(t_{0}-a\right)=x\left(t_{0}+b\right)$.

Taking these uncertainties into account, the next step in the analysis is to evaluate their impact on the envelopes $e_{\min }(t)$ and $e_{\max }(t)$. Usually, these envelopes are computed using a cubic spline interpolation $[1,3]$. In our model however, we will use piecewise linear interpolation (this is the only simplification we consider for the elementary operator $\mathcal{S}$ ). The reason for this is simply that the value of a cubic spline interpolation between two knots depends not only on a few knots around but also on all the knots defining the interpolation. In this context, calculating an uncertainty on the value of the interpolation at a specific position, given uncertainties on all the knots, is rather complicated. It turns out that evaluation is much simpler with a piecewise linear interpolation, while guaranteeing that the obtained uncertainties generally match the observations.

If we then consider two consecutive maxima $\left(t_{0}, x\left(t_{0}\right)\right)$ and $\left(t_{1}, x\left(t_{1}\right)\right)$ in the continuous-time signal, the corresponding maxima in the sampled signal are located within the rectangular boxes $\left\{\left[t_{i}-\Delta_{a b s .}^{(i)}, t_{i}+\Delta_{a b s .}^{(i)}\right] \times\left[x\left(t_{i}\right)-\Delta_{o r d .}^{(i)}, x\left(t_{i}\right)\right] ; i=0,1\right\}$ (see Figure 3). In this context, it is clear that the largest error for the envelope is obtained for the thick dash-dot line case. Integrating this error over the range $\left[t_{0}, t_{1}\right]$ (corresponding to the shaded surface in Figure 3 ), we get over this range a bound (referred to as $\delta e_{\max }(t)$ ) on the sampling error for the upper envelope $e_{\max }(t)$ :

$\int_{t_{0}}^{t_{1}}\left|\delta e_{\max }(t)\right| d t \leq \frac{\left(t_{1}-t_{0}\right)\left(\Delta_{\text {ord. }}^{(0)}+\Delta_{\text {ord. }}^{(1)}\right)}{2}+\left(t_{1}-t_{0}\right) \frac{\left|x\left(t_{1}\right)-x\left(t_{0}\right)-\Delta_{\text {ord. }}^{(1)}+\Delta_{\text {ord. }}^{(0)}\right|\left(\Delta_{\text {abs. }}^{(1)}+\Delta_{\text {abs. }}^{(0)}\right)}{2\left(t_{1}-t_{0}-\left|\Delta_{\text {abs. }}^{(1)}-\Delta_{\text {abs. }}^{(0)}\right|\right)}$,

which leads in turn to the looser bound:

$$
\begin{aligned}
& \int_{t_{0}}^{t_{1}}\left|\delta e_{\max }(t)\right| d t \leq\left(t_{1}-t_{0}\right) \cdot \frac{\Delta_{\text {ord. }}^{(0)}+\Delta_{\text {ord. }}^{(1)}}{2}+\left|x\left(t_{1}\right)-x\left(t_{0}\right)\right| \cdot \frac{\Delta_{a b s .}^{(1)}+\Delta_{a b s .}^{(0)}}{2} \\
+ & \frac{t_{1}-t_{0}}{t_{1}-t_{0}-\left|\Delta_{\text {abs. }}^{(1)}-\Delta_{\text {abs. }}^{(0)}\right|} \cdot \frac{\Delta_{a b s .}^{(1)}+\Delta_{a b s .}^{(0)}}{2} \cdot\left|\Delta_{\text {ord. }}^{(1)}-\Delta_{\text {ord. }}^{(0)}\right|+\frac{\left|\Delta_{a b s .}^{(1)}-\Delta_{a b s .}^{(0)}\right|}{t_{1}-t_{0}-\left|\Delta_{a b s .}^{(1)}-\Delta_{a b s .}^{(0)}\right|} \cdot\left|x\left(t_{1}\right)-x\left(t_{0}\right)\right|,
\end{aligned}
$$

In the last formula, the first term can be read as the average uncertainty caused by the uncertainties in ordinate only, its amplitude depending only on the behaviour of the signal around 


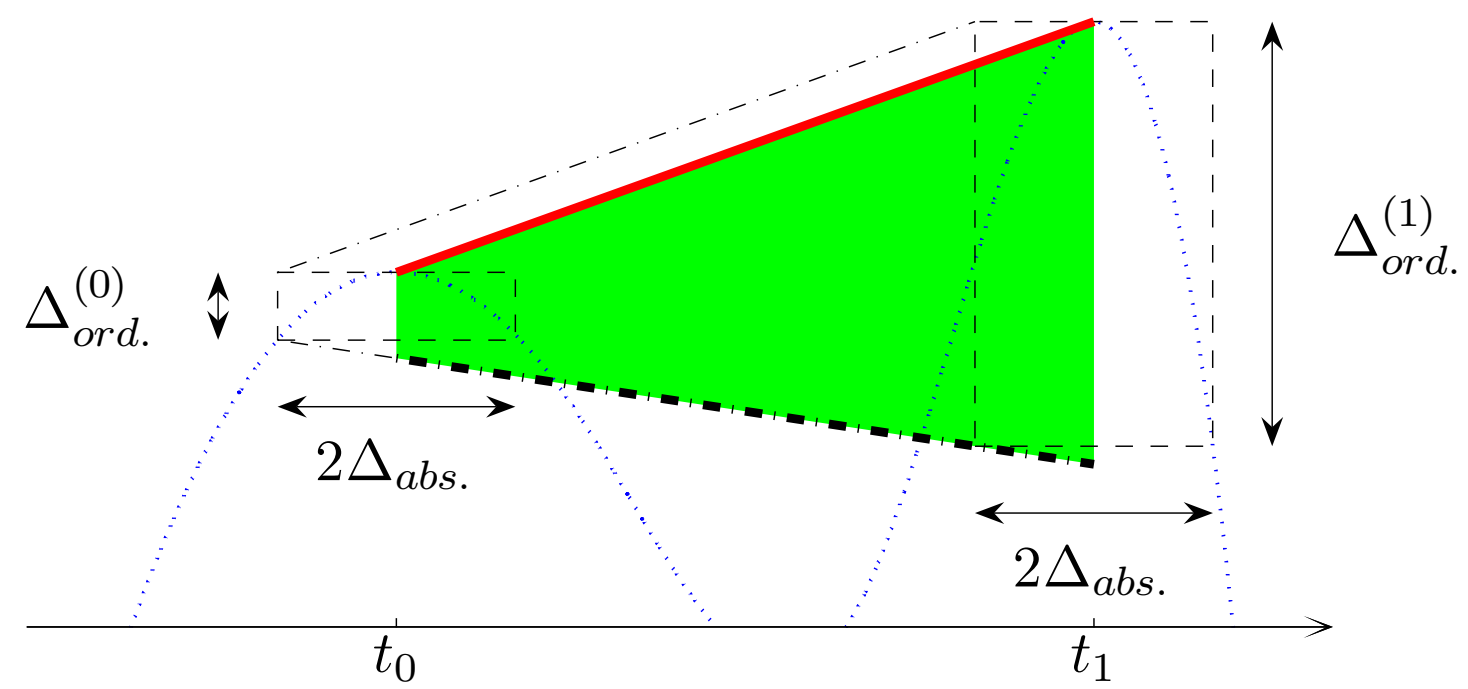

Figure 3: Evaluating the uncertainty for the envelopes. For both maxima, the dashed boxes delimit the areas where the corresponding maxima in the sampled signal can be located. The thick plain line stands for the interpolation (piecewise linear in the model) based on the extrema in the continuous-time signal. Then the dash-dot lines delimit the area where the interpolation based on the extrema in the sampled signal can be located. Finally, the thick dash-dot line stands for the case that leads to the largest error.

its extrema. This contrasts with the second term which, depending on the uncertainties in abscissa, does not only depend on the behaviour of the signal around its extrema, but also on the relative amplitudes of successive extrema. If the analyzed signal is, e.g., an amplitude modulated (AM) sinusoidal signal, then the amplitude of the first term will only depend on the sampling frequency and on the frequency of the AM signal, while the second one will depend on the frequency and amplitude of the modulation too. Finally, the last terms, depending on both uncertainties, are generally much smaller than the first two ones and can therefore be neglected in most cases.

If we compute the bound (11) on each range delimited by two consecutive maxima, we obtain a bound for the $L_{1}$-norm of the sampling related effects on the upper envelope. Repeating the same operation for the lower envelope then results in a bound on the error for the mean of the envelopes, which is simply half the sum of bounds on each envelope. Finally, this bound is also valid for the elementary operator defined as the difference between the signal and the mean of the envelopes.

\subsubsection{Estimating the parameters from the signal}

Given a signal $x(t)$, computing the upper bound (11) requires only a limited set of parameters (namely, the positions $\left(t_{i}, x\left(t_{i}\right)\right)$ and the uncertainties $\Delta_{a b s .}^{(i)}$ and $\Delta_{o r d}^{(i)}$ for each extremum) that are rather easy to measure in continuous-time. However, if we only have a discrete version of the signal, some of these parameters cannot be evaluated without prior additional information. 
Indeed, the $\left(t_{i}, x\left(t_{i}\right)\right)$ can be estimated by the corresponding extrema values in the discrete signal: $\hat{t}_{i}=n_{i} / f_{s}+\varphi$ and $\hat{x}_{i}=\widehat{x\left(t_{i}\right)}=x_{f_{s}, \varphi}\left[n_{i}\right]$, where $n_{i}$ is the index of the $i^{\text {th }}$ extremum in the discrete signal. In these estimates the time instants $t_{i}$ are estimated with a precision of order $1 / f_{s}$. This unfortunately implies that the uncertainties in abscissa $\Delta_{a b s .}^{(i)} \in\left[1 / 2 f_{s}, 1 / f_{s}[\right.$ cannot be estimated with a decent precision, thus constraining us to use the upper bound $1 / f_{s}$ as a default value for all extrema. Concerning the $\Delta_{o r d}^{(i)}$. however, these can be estimated but only if we have some additional information on the behaviour of the signal around its extrema. A natural way to proceed is to make a regularity assumption on the signal: if we, e.g., require the signal to be twice continuously differentiable, then we can use a second order Taylor expansion to estimate the behaviour of the signal around its extrema. We can this way use the parabolic approximation $x(t) \approx \frac{1}{2}\left(t-t_{i}\right)^{2} x^{\prime \prime}\left(t_{i}\right)$, which leads to $\Delta_{\text {ord. }}^{(i)} \approx \frac{1}{2}\left|x^{\prime \prime}\left(t_{i}\right)\right| / f_{s}^{2}$. Finally, the second derivatives $x^{\prime \prime}\left(t_{i}\right)$ can themselves be estimated using finite difference operators at the extrema of the sampled signal, leading to $\hat{x}_{i}^{\prime \prime}$. Putting these approximations back into (11) results in an estimate of the maximum sampling error over the range $\left[t_{i}, t_{i+1}\right]$ for the upper envelope:

$$
\int_{t_{i}}^{t_{i+1}}\left|\delta e_{\max }(t)\right| d t \leq \frac{\left(\hat{t}_{i+1}-\hat{t}_{i}\right)\left|\hat{x}_{i}^{\prime \prime}+\hat{x}_{i+1}^{\prime \prime}\right|}{4 f_{s}^{2}}+\frac{\left|\hat{x}_{i+1}-\hat{x}_{i}\right|}{f_{s}}+\frac{\left|\hat{x}_{i+1}^{\prime \prime}-\hat{x}_{i}^{\prime \prime}\right|}{2 f_{s}^{3}} .
$$

We are thus led to the central result of this paper, i.e., the existence of an upper bound for the $L_{1}$-norm of the sampling error for the elementary operator $\delta \mathcal{S}$ of the form:

$$
\|\delta \mathcal{S} x\|_{1} \leq \frac{\lambda}{f_{s}}+\frac{\mu}{f_{s}^{2}}+\frac{\nu}{f_{s}^{3}},
$$

where the integration for the $L_{1}$-norm essentially extends to the observation interval of the analyzed signal and with

$$
\begin{aligned}
\lambda & =\frac{1}{2}\left(\sum_{i}\left|\hat{x}_{i+1}^{m}-\hat{x}_{i}^{m}\right|+\sum_{i}\left|\hat{x}_{i+1}^{M}-\hat{x}_{i}^{M}\right|\right), \\
\mu & =\frac{1}{8}\left(\sum_{i}\left(\hat{t}_{i+1}^{m}-\hat{t}_{i}^{m}\right)\left|\hat{x}_{i}^{m \prime \prime}+\hat{x}_{i+1}^{m \prime \prime}\right|+\sum_{i}\left(\hat{t}_{i+1}^{M}-\hat{t}_{i}^{M}\right)\left|\hat{x}_{i}^{M \prime \prime}+\hat{x}_{i+1}^{M \prime \prime}\right|\right), \\
\nu & =\frac{1}{4} \sum_{i}\left|\hat{x}_{i+1}^{m \prime \prime}-\hat{x}_{i}^{m \prime \prime}\right|+\left|\hat{x}_{i+1}^{M \prime \prime}-\hat{x}_{i}^{M \prime \prime}\right|,
\end{aligned}
$$

where superscripts $m$ and $M$ refer to minima and maxima, respectively.

Remark - Our previous studies showed that, in the specific case of sinusoidal signals, the sampling error is upper bounded by a function proportional to $f_{s}^{-2}$ [4]. The bound (12) obtained here generalizes this result since the parameters $\lambda$ and $\nu$ are simply zero in the sinusoidal case. Nevertheless, the $\mu$ coefficient computed with (14) for sinusoidal signals leads to a bound that is looser than the one we obtained in [4], and there are two reasons for this. First, a sinusoidal signal is symmetric with respect to each extremum: therefore, the uncertainty in abscissa $\Delta_{a b s}$. can be reduced to $1 /\left(2 f_{s}\right)$, leading to the reduced parameter values: $\lambda^{\prime}=\lambda / 2, \mu^{\prime}=\mu / 4$ and $\nu^{\prime}=\nu / 8$. 
Second, there is generally a partial compensation between the errors associated to the $f_{s}^{-2}$ terms coming from the upper and the lower envelope. This can be easily controlled for the sinusoidal case, allowing a bound reduced by one half and thus in agreement with our former results.

\subsubsection{Validation}

To assess the performance of the upper bound model (12), we need to measure the difference between the continuous-time EMD of a continous-time signal and the discrete-time EMDs of sampled versions of the same signal for various sampling parameters. To that end, we performed a set of simulations using synthetic piecewise polynomial signals. As the extrema of a polynomial of order less than 4 are analytically known, the continuous-time EMD of a piecewise polynomial can be defined properly. In a first time however, we will only study differences between the discrete and continuous-time elementary operators, as for the model, and the overall methodology can be summarized by the following procedure:

1. synthetize a continuous-time piecewise polynomial oscillating signal $x(t), t \in[0, L]$ such that the minimum distance between two extrema is greater than 2 , and $L$ is such that the number of extrema is large enough to neglect border effects.

2. apply the continuous-time elementary operator: $y_{\infty}(t) \equiv(\mathcal{S} x)(t)$.

3. define the sampled signals: $x_{f_{s}, \varphi}[n] \equiv x\left(\frac{n}{f_{s}}+\varphi\right)$ for $0 \leq n \leq N\left(f_{s}\right) \equiv\left\lfloor L f_{s}\right\rfloor-1$ and $0 \leq \varphi<\frac{1}{f_{s}}$ (where $\lfloor\cdot\rfloor$ stands for the integer part).

4. apply the elementary sifting operator to each sampled signal: $y_{f_{s}, \varphi}[n] \equiv\left(\mathcal{S} x_{f_{s}, \varphi}\right)[n]$.

5. for each sampling parameters set $\left(f_{s}, \varphi\right)$, compute the sampling error measure ${ }^{4}$ :

$$
e\left(f_{s}, \varphi\right) \equiv \frac{1}{N\left(f_{s}\right)+1} \sum_{n=0}^{N\left(f_{s}\right)}\left|y_{f_{s}, \varphi}[n]-y_{\infty}\left(\frac{n}{f_{s}}+\varphi\right)\right| .
$$

The test signals we used are piecewise cubic polynomials obtained through interpolation of a random set of extrema. The main property of the underlying model is that it ensures that the minimum distance between extrema is set by a parameter $\Delta$. The details are of lesser importance as the results seem to be weakly dependent on the model.

Simulation results are plotted in Figure 4 for two representative examples. As it can be seen on the figure, the behaviour of the bound as a function of the sampling frequency can generally be divided in two areas: for lower sampling frequencies, the bound usually behaves like $f_{s}^{-2}$ whereas, for higher frequencies, it behaves like $f_{s}^{-1}$ (see Figure 4-(b)). There are specific cases however where the $f_{s}^{-1}$ area does not exist, since the coefficient $\lambda$ given by (13) is typically zero when all the maxima/minima have the same amplitude (see Figure 4-(a)). This twofold behaviour also applies

\footnotetext{
${ }^{4}$ The choice made here of a $l_{1}$-norm is not critical, other norms leading to similar results.
} 
to the measured sampling error, but either the $f_{s}^{-1}$ or the $f_{s}^{-2}$ area can be missing. Moreover, when both are present, the critical sampling frequency delimiting these areas is generally not the same as the corresponding critical frequency for the bound. The evolution of the bound however usually gives a good outline for the evolution of the sampling error. Besides, the $f_{s}^{-3}$ behaviour that we could expect from (12) has never been observed distinctly.

Quantitatively, the sampling error measured in our simulations is always rather far from the bound. The latter usually is around $12 \mathrm{~dB}$ above in the $f_{s}^{-1}$ area and $25 \mathrm{~dB}$ in the $f_{s}^{-2}$ area. This discrepancy comes mainly from the fact that the bound is obtained by considering a worst case error everywhere whereas this worst case is rather improbable. To relate the bound to the simulation results, we can consider a variation on the model giving an average value instead of an upper bound for the errors in abscissa and ordinate for each extremum. Basically, we can take averaged values of the errors with respect to the sampling phase:

$$
\begin{aligned}
\forall i, \quad \bar{\Delta}_{\text {abs. }}^{(i)} & =\mathbb{E}_{\varphi}\left\{\left|t_{i}-\left(n_{i} f_{s}+\varphi\right)\right|\right\}, \\
\bar{\Delta}_{\text {ord. }}^{(i)} & =\mathbb{E}_{\varphi}\left\{\left|x\left(t_{i}\right)-x_{f_{s}, \varphi}\left[n_{i}\right]\right|\right\},
\end{aligned}
$$

where $n_{i}$ refers to the index of the extremum corresponding to $\left(t_{i}, x\left(t_{i}\right)\right)$ in the discrete signal. In order to estimate these quantities, we can use the local parabolic approximation again, thus leading to:

$$
\begin{aligned}
& \bar{\Delta}_{a b s .}^{(i)}=\frac{\Delta_{a b s .}^{(i)}}{2}, \\
& \bar{\Delta}_{\text {ord. }}^{(i)}=\frac{\Delta_{\text {ord } .}^{(i)}}{3} .
\end{aligned}
$$

Now, even with this average model, the sampling error estimate still lies several $\mathrm{dB}$ above the simulation results. One reason for this is that we use $1 / f_{s}$ for the uncertainties in abscissa while we know that the actual uncertainty with our test signals is generally smaller than $0.7 / f_{s}$. If we had used an average value $\alpha / f_{s}, 1 / 2 \leq \alpha<1$ (instead of $1 / f_{s}$ ), then the three parameters $\lambda, \mu$ and $\nu$ defined by equations (13), (14) and (15) would have been replaced by:

$$
\lambda^{\prime}=\alpha \lambda, \quad \mu^{\prime}=\alpha^{2} \mu \quad \text { and } \quad \nu^{\prime}=\alpha^{3} \nu .
$$

Combining these with the former average model, we get the following estimate for the average sampling error $(A S E)$ :

$$
A S E=\frac{\alpha \lambda}{2}+\frac{\alpha^{2} \mu}{3}+\frac{\alpha^{3} \nu}{6} .
$$

The average model with corrected uncertainties in abscissa is much closer to the simulation results but still some $\mathrm{dB}$ above. There are mainly two reasons for the remaining error. First, there is a deviation coming from the use of a parabolic approximation around the extrema to compute the uncertainties in ordinate. Second, the sampling error on the upper envelope generally 

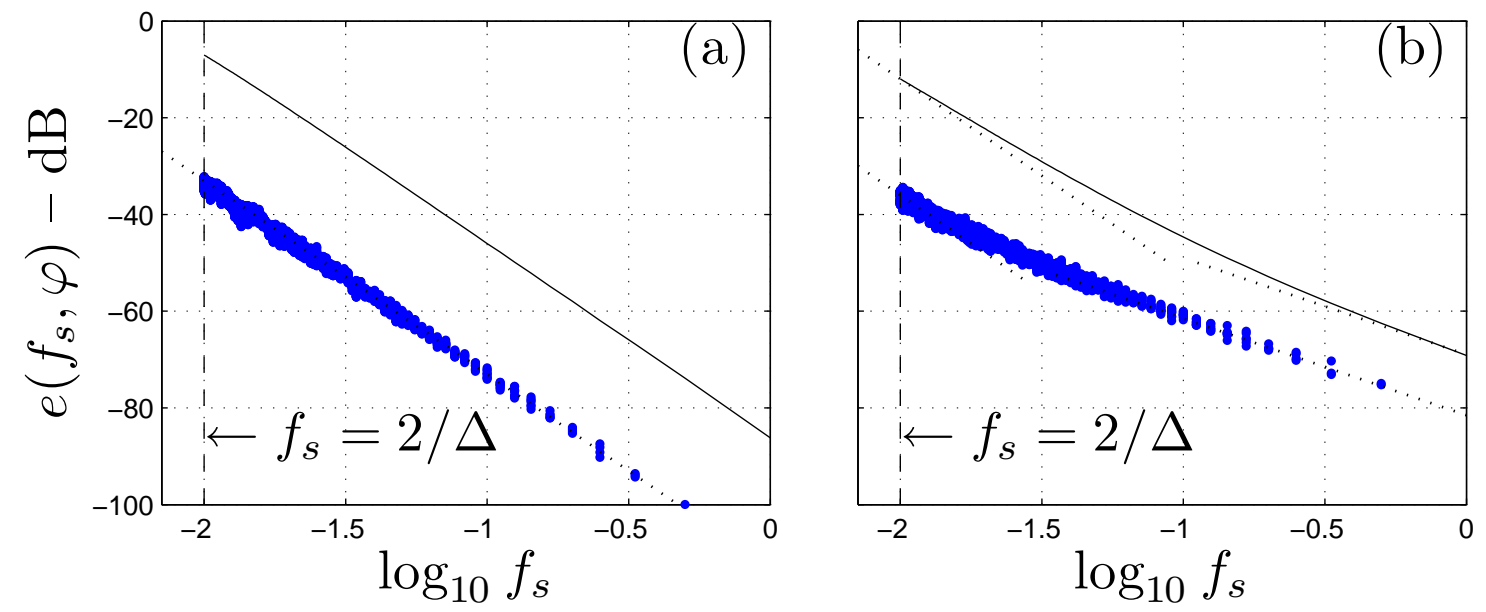

Figure 4: Sampling error and bound estimate as a function of the sampling frequency. On both graphs, each dot stands for a measure of sampling error according to (16). For each sampling frequency and phase, a bound is also estimated according to (12) from the downsampled signal: the mean of these with respect to the phase is plotted against sampling frequency as a full line. Cases (a) and (b) correspond to rather close signals, the difference being that (a) has constant maxima/minima amplitudes while (b) has random maxima/minima values centered around 1/-1 with variance 0.1 . Asymptotic $f_{s}^{-1}$ or $f_{s}^{-2}$ behaviours are evidenced by dotted lines.

partially (sometimes totally) compensates for the sampling error on the lower envelope, whereas the two corresponding bounds are just summed in the model. Unfortunately, the magnitude of the compensation cannot be estimated properly from the discrete signal. Indeed, it locally depends on the fractional part of the product of the sampling frequency by the distance between a maximum and the next minimum in the continuous-time signal. However, the precision on the estimation of the distance between a maximum and the next minimum is roughly $1 / f_{s}$ and, therefore, the corresponding precision on the fractional part of the previous product is roughly 1 , which is insufficient.

\section{Conclusion}

The question of the influence of sampling on EMD has been addressed here in some detail, from the derivation of theoretical results in simplified cases to the observation of effective behaviours in more realistic situations. The existence of bounds on possible errors due to sampling allows now for a quantitative approach which goes beyond the qualitative precautionary principle of only applying EMD to "sufficiently oversampled" data. A number of questions are still left open, among which stands first the analysis of what actually happens when iterating sifting as it is done in practice, with the possible appearance of new extrema pairs within the process. Such a point is under current investigation and will be reported elsewhere. 


\section{References}

[1] N. E. Huang, Z. Shen, S. R. Long, M. L. Wu, H. H. Shih, Q. Zheng, N. C. Yen, C. C. Tung and H. H. Liu, "The empirical mode decomposition and Hilbert spectrum for nonlinear and non-stationary time series analysis," Proc. Roy. Soc. London A, Vol. 454, pp. 903-995, 1998.

[2] N. E. Huang, M. L. C. Wu, S. R. Long, S. S. P. Shen, W. D. Qu, P. Gloersen, and K. L. Fan, "A confidence limit for the empirical mode decomposition and Hilbert spectral analysis," Proc. Roy. Soc. London A, Vol. 459, pp. 2317-2345, 2003.

[3] G. Rilling, P. Flandrin and P. Gonçalvès, "On Empirical Mode Decomposition and its Algorithms," in Proc. IEEE-EURASIP Workshop on Nonlinear Signal and Image Processing NSIP-03, Grado (I), 2003.

[4] G. Rilling and P. Flandrin, "Sur la décomposition modale empirique des signaux échantillonnés," in Proc. 20 Coll. GRETSI sur le Traitement du Signal et des Images, pp. 755-758, Louvain-la-Neuve (B), 2005.

[5] G. Rilling and P. Flandrin, "On the influence of sampling on the Empirical Mode Decomposition," in Proc. IEEE Int. Conf. on Acoust., Speech and Signal Proc. ICASSP-06, Toulouse (F), 2006 (to appear).

[6] N. Stevenson, M. Mesbah and B. Boashash, "A sampling limit for the Empirical Mode Decomposition," in Proc. Int. Symp. on Signal Proc. and its Appl. ISSPA-05, pp. 647-650, Sydney (A), 2005.

[7] http://perso.ens-lyon.fr/patrick.flandrin/emd.html. 